\title{
Performance assessment of different pulse reconstruction algorithms for the ATHENA X-ray Integral Field Unit
}

Peille, Philippe, Ceballos, Maria Teresa, Cobo, Beatriz, Wilms, Joern, Bandler, Simon, et al.

Philippe Peille, Maria Teresa Ceballos, Beatriz Cobo, Joern Wilms, Simon Bandler, Stephen J. Smith, Thomas Dauser, Thorsten Brand, Roland den Hartog, Jelle de Plaa, Didier Barret, Jan-Willem den Herder, Luigi Piro, Xavier Barcons, Etienne Pointecouteau, "Performance assessment of different pulse reconstruction algorithms for the ATHENA X-ray Integral Field Unit," Proc. SPIE 9905, Space Telescopes and Instrumentation 2016: Ultraviolet to Gamma Ray, 99055W (11 July 2016); doi: 10.1117/12.2232011

Event: SPIE Astronomical Telescopes + Instrumentation, 2016, Edinburgh, United Kingdom 


\title{
Performance assessment of different pulse reconstruction algorithms for the Athena X-ray Integral Field Unit
}

\author{
Philippe Peille ${ }^{\mathrm{a}}$, Maria Teresa Ceballos ${ }^{\mathrm{b}}$, Beatriz Cobo ${ }^{\mathrm{b}}$, Joern Wilms ${ }^{\mathrm{c}}$, Simon Bandler ${ }^{\mathrm{d}}$, \\ Stephen J. Smith ${ }^{\mathrm{d}}$, Thomas Dauser ${ }^{\mathrm{c}}$, Thorsten Brand ${ }^{\mathrm{c}}$, Roland den Hartoge ${ }^{\mathrm{e}}$, Jelle de Plaa , \\ Didier Barret ${ }^{\mathrm{a}}$, Jan-Willem den Herder ${ }^{\mathrm{e}}$, Luigi Piro ${ }^{\mathrm{f}}$, Xavier Barcons ${ }^{\mathrm{b}}$, and Etienne \\ Pointecouteau $^{\mathrm{a}}$

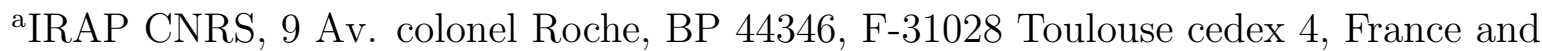 \\ Université de Toulouse III Paul Sabatier / OMP, Toulouse, France \\ bInstituto de Física de Cantabria (CSIC-UC), Edificio Juan Jordá, Avenida de los Castros, s/n \\ - E-39005 Santander, Cantabria \\ cECAP, University of Erlangen-Nuremberg Sternwartstr. 796049 Bamberg, Germany \\ dNASA/Goddard Space Flight Center, 8800 Greenbelt Rd, Greenbelt, MD 20771, United \\ States \\ eSRON, Netherlands Institute for Space Research, Sorbonnelaan 2, 3584 CA Utrecht \\ ${ }^{\mathrm{f}}$ Istituto di Astrofisica e Planetologia Spaziali, Via Fosso del Cavaliere 100, 00133, Roma, Italy
}

\begin{abstract}
The X-ray Integral Field Unit (X-IFU) microcalorimeter, on-board Athena, with its focal plane comprising 3840 Transition Edge Sensors (TESs) operating at $90 \mathrm{mK}$, will provide unprecedented spectral-imaging capability in the $0.2-12 \mathrm{keV}$ energy range. It will rely on the on-board digital processing of current pulses induced by the heat deposited in the TES absorber, as to recover the energy of each individual events. Assessing the capabilities of the pulse reconstruction is required to understand the overall scientific performance of the X-IFU, notably in terms of energy resolution degradation with both increasing energies and count rates. Using synthetic data streams generated by the X-IFU End-to-End simulator, we present here a comprehensive benchmark of various pulse reconstruction techniques, ranging from standard optimal filtering to more advanced algorithms based on noise covariance matrices. Beside deriving the spectral resolution achieved by the different algorithms, a first assessment of the computing power and ground calibration needs is presented. Overall, all methods show similar performances, with the reconstruction based on noise covariance matrices showing the best improvement with respect to the standard optimal filtering technique. Due to prohibitive calibration needs, this method might however not be applicable to the X-IFU and the best compromise currently appears to be the so-called resistance space analysis which also features very promising high count rate capabilities.
\end{abstract}

Keywords: Athena, X-IFU, X-rays, microcalorimeters, pulse reconstruction, performance analysis

\section{INTRODUCTION}

Scheduled for launch in 2028 on board the Athena X-ray observatory, the X-ray Integral Field Unit (X-IFU $\left.{ }^{1}\right)$ will provide spectral-imaging capability in the $0.2-12 \mathrm{keV}$ with 5 " spatial resolution and an unprecedented $2.5 \mathrm{eV}$ energy resolution at $7 \mathrm{keV}$. It will address a variety of key scientific questions such as the dynamics and enrichment of clusters of galaxies, or the composition of the Warm Hot Intergalactic Medium. ${ }^{2}$ The X-IFU is a cryogenic micro-calorimeter whose focal plane will be populated by 3840 X-ray absorbers organized following a hexagonal pattern across a 5' diameter field of view. Each absorber will be thermally linked to a Transition Edge Sensor (TES) voltage biased in its transition between the superconducting and normal states. The thermalization of an X-ray in the absorber will thus induce a sharp change of the TES resistance which will be measured as a

Further author information: (Send correspondence to Philippe Peille)

E-mail: philippe.peille@irap.omp.eu

Space Telescopes and Instrumentation 2016: Ultraviolet to Gamma Ray, edited by

Jan-Willem A. den Herder, Tadayuki Takahashi, Marshall Bautz, Proc. of SPIE Vol. 9905, 99055W

(c) 2016 SPIE · CCC code: 0277-786X/16/\$18 - doi: 10.1117/12.2232011

Proc. of SPIE Vol. $990599055 \mathrm{~W}-1$ 

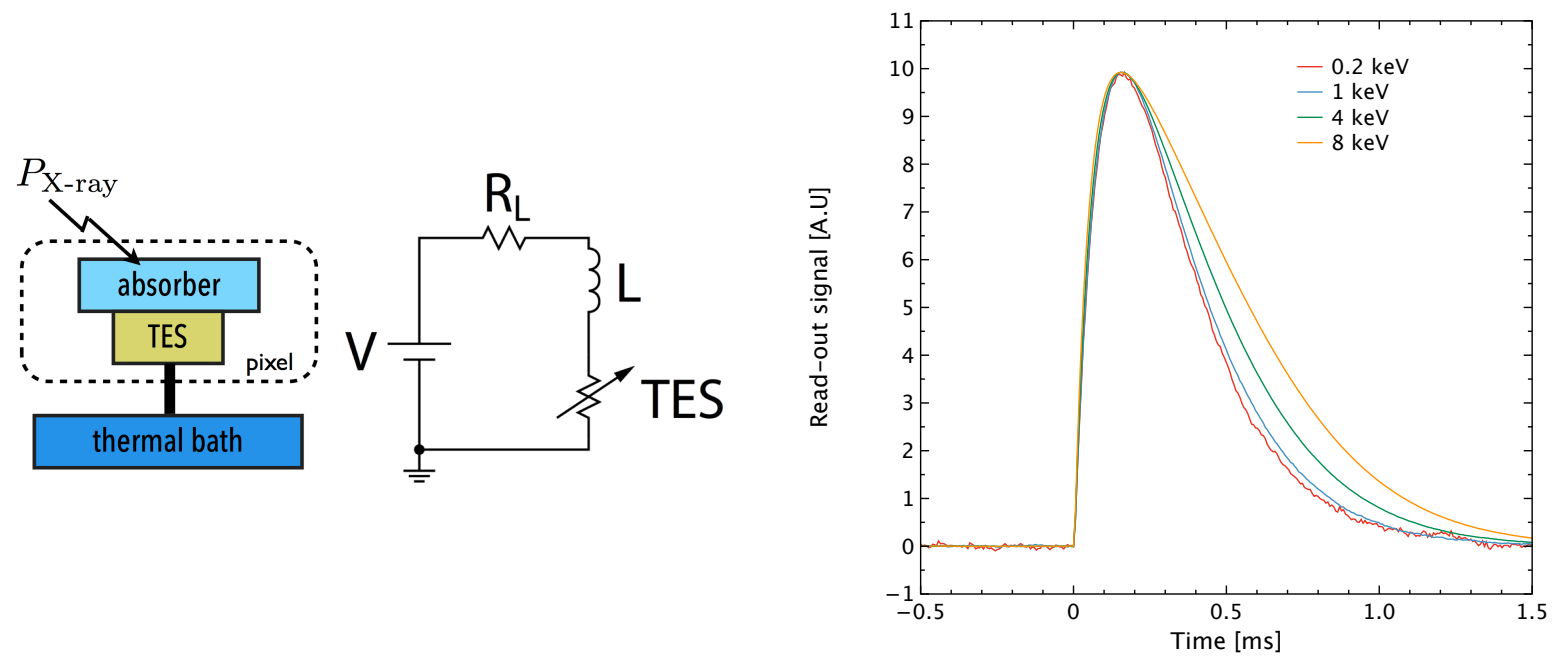

Figure 1. Left: Schematics of the physics model coupling the thermal and electrical behavior of the TES/absorber pixel used by tessim. For the thermal part, the link to the heat bath, the absorption of X-ray photons and the Joule dissipation are simulated. On the electrical side, an equivalent circuit with a constant voltage bias, a load resistor, an inductance and the variable TES resistant is used. Right: Example of simulated current pulses at different energies. The signals were flipped and scaled to have the same peak value to illustrate the simulation non-linearities.

pulse in the TES current. In order to limit the heat load on the cryogenic chain, the TESs will not be read out independently but pooled in 96 frequency multiplexed channels (FDM). ${ }^{3}$ At the end of the readout chain, an on-board Event Processor (EP) will be responsible for the extraction of the time and energy information from the measured current pulses. Assessing the capabilities of the pulse reconstruction is therefore key to understand the overall performance of the instrument.

Over the past few years, several new pulse reconstruction techniques have been proposed to improve and replace the now widely used optimal filtering technique. ${ }^{4-6}$ The main motivation for this is to reduce the energy resolution degradation with energy observed in non-linear detectors such as the TESs. In this paper, we will present a comprehensive benchmark of these analysis methods considered for the read out of the X-IFU pixels using a TES simulator $\left(\right.$ tessim $\left.^{7}\right)$ newly developed in the frame of the X-IFU End-to-End simulator SIXTE. ${ }^{8}$

After presenting the physics model used by tessim (Sect. 2), we will review the different tested pulse processing algorithms (Sect. 3) and discuss their performance not only in terms of energy resolution degradation at high energy, but also in terms of count rate capability (Sect. 4). Finally, we will give a first comparative assessment of the computing power (Sect. 5) and ground calibration needs (Sect. 6) of theses techniques.

\section{SIMULATING X-IFU PIXEL READOUT STREAMS WITH TESSIM}

tessim is based on a generic model of the TES/absorber pixel considered as a single thermodynamical element interacting with a cold bath and an X-ray loading, together with its first stage read-out circuit including a voltage bias, a load resistor and an inductance (see e.g. Mather et al., 1982). ${ }^{9}$ The overall setup of the model is presented in Figure 1 (left) and details on this software are available in Wilms et al. (2016). ${ }^{7}$ In brief, the code performs a numerical solution of the differential equations for the time-dependent temperature, $T(t)$, and current, $I(t)$, in the TES, using the formulation of Irwin \& Hilton $(2005):{ }^{10}$

$$
\begin{aligned}
C \frac{d T}{d t} & =-P_{\mathrm{b}}+R(T, I) I^{2}+P_{\mathrm{X}-\text { ray }}+\text { Noise } \\
L \frac{d I}{d t} & =V_{0}-I R_{\mathrm{L}}-I R(T, I)+\text { Noise. }
\end{aligned}
$$

where $C$ is the heat capacity of the pixel, $P_{b}$ the power flow to the heat bath and $P_{\mathrm{X} \text {-ray }}$ is the power deposited by X-rays thermalizing in the absorber. In the electrical equation, $L$ is the effective inductance of the read-out 
Table 1. Pixel parameters used to simulate the baseline X-IFU pixels.

\begin{tabular}{|l|l|}
\hline Pixel parameter & Value \\
\hline Heat capacity at bias $C$ & $0.8 \mathrm{pJ} / \mathrm{K}$ \\
Bath conductance at bias & $200 \mathrm{pW} / \mathrm{K}$ \\
Heat bath power flow exponent & 3 \\
$\alpha$ & 75 \\
$\beta$ & 1.25 \\
Bias resistance $R_{0}$ & $1 \mathrm{~m} \Omega$ \\
Bias temperature $T_{0}$ & $90 \mathrm{mK}$ \\
Bias current $I_{0}$ & $68 \mu \mathrm{A}$ \\
Effective load resistance $R_{L}$ & $49 \mu \Omega$ \\
Effective circuit inductance $L$ & $66 \mathrm{nH}$ \\
Read-out sampling frequency & $156.25 \mathrm{kHz}$ \\
\hline
\end{tabular}

circuit, $R_{L}$ the effective load resistor, and $V_{0}$ the constant voltage bias. To model the TES transition, tessim uses a simple temperature and current dependent resistance plane:

$$
R(T, I)=R_{0}+\left.\frac{\partial R}{\partial T}\right|_{I_{0}}\left(T-T_{0}\right)+\left.\frac{\partial R}{\partial I}\right|_{T_{0}}\left(I-I_{0}\right)
$$

where the partial derivatives are defined at the TES bias point with the parameters

$$
\begin{gathered}
\alpha=\left.\frac{\partial \log R}{\partial \log T}\right|_{I_{0}}=\left.\frac{T_{0}}{R_{0}} \frac{\partial R}{\partial T}\right|_{I_{0}}, \\
\beta=\left.\frac{\partial \log R}{\partial \log I}\right|_{T_{0}}=\left.\frac{I_{0}}{R_{0}} \frac{\partial R}{\partial I}\right|_{T_{0}} .
\end{gathered}
$$

The link to the thermal bath is modeled following the prescription of Irwin \& Hilton (2005). ${ }^{10}$ Currently, tessim simulates the following noise sources: thermal fluctuations between the TES and the heat bath, electrical Johnson noise in the TES and shunt resistor, as well as readout noise from the SQUID and amplifier chain. All noise levels are recomputed throughout the simulation such that their non-stationary character is respected. The Johnson noise notably significantly decreases during a pulse.

In order to simulate representative X-IFU pixels, we took pixel parameters adapted from the canonical pixels of the X-ray Microcalorimeter Spectrometer studied for the International X-ray Observatory, ${ }^{11}$ which had similar energy resolution and count rate requirements to the X-IFU. The adopted values are summarized in Table 1 (see Smith et al., $2016^{12}$ for more details). Figure 1 (right) shows sample pulses simulated with these pixels at different energies. One can notably notice how the pulse shape changes with energy due to the non-linearities of the detector and its read-out circuit. In order to simplify our analysis, all simulated pulses will be in phase with the read-out process such that no particular care will be needed for corrections of pulse arrival time effects.

\section{PRESENTATION OF THE DIFFERENT PULSE RECONSTRUCTION TECHNIQUES}

\subsection{The standard optimal filtering technique}

First introduced by Szymkowiak et al. (1993) ${ }^{4}$ optimal filtering has rapidly become the standard technique for the analysis of X-ray micro-calorimeter pulses and has been used by all the currently flown instruments. ${ }^{5,6}$ In brief, it consists of a $\chi^{2}$ fit of the pulses in the frequency domain assuming that every pulse is an energy scaled 
version of a single template $(d(t)=E \times s(t))$ and weighting by the measured noise spectrum of the system (assumed to be stationary):

$$
\chi^{2}=\sum \frac{[D(f)-E \times S(f)]^{2}}{N^{2}(f)},
$$

where $D(f)$ and $S(f)$ are the Discrete Fourier Transforms of the signal and template, and $N^{2}(f)$ the power spectrum of the noise measured from empty streams. As Equation 6 is a linear $\chi^{2}$ problem, the optimal energy estimator $\hat{E}$ can be computed with a single dot product, whose time domain formula is given by:

$$
\hat{E}=\frac{\left\langle d(t), \mathcal{F} \mathcal{F} \mathcal{T}^{-1}\left\{S(f) / N^{2}(f)\right\}\right\rangle}{\sum|S(f)|^{2} / N^{2}(f)} .
$$

Usually, because of the presence of significant $1 / f$ noise in the data stream due for instance to fluctuations of the TES thermal environment, the $0 \mathrm{~Hz}$ bin of the Fourier Transform is not used, such that the final filter is zero summed and the signal baseline is effectively rejected from the processing.

Of course, the assumption of a single pulse shape is not verified in non-linear detectors such as the X-IFU pixels. As a consequence, the raw energy estimation $\hat{E}$ has to be transformed to an unbiased final estimation $\hat{E}_{\text {final }}$ by the application of a gain scale typically obtained from the filtering of pulse templates measured at different energies. In the work presented here, we used $1 \mathrm{keV}$ pulse shapes obtained from the averaging of many simulated streams, but our results were found to be mainly independent of this choice. It is important to note that even if the optimal filter is an unbiased estimate of the pulse energy when using a template computed at the exact same energy, any measure of the corresponding spectral resolution needs to take into account the associated gain scale in order to be in physical units.

\section{2 "Resistance space" analysis}

In order to treat the intrinsic non-linearities of the optimal filtering method, one can initially transform the input signal before the reconstruction such that it presents a more linear scaling with energy and a more stationary noise. Some authors notably proposed the so-called "resistance space" analysis which uses a proxy to the resistance signal instead of the current to perform the optimal filtering. ${ }^{13,14}$ The idea is to suppress the main source of non-linearity of the detectors which comes from the first stage read-out circuit. For this transformation, the circuit inductance was neglected in previous works ${ }^{14}$ and in the case of our model (see Fig. 1, left) this would translate to:

$$
\tilde{R}(t)=\frac{V_{0}-I(t) R_{L}}{I(t)} .
$$

In Figure 2, we compare the transformed $7 \mathrm{keV}$ pulse shape to the simulated resistance going through the TES as well as the resulting gain scale obtained by applying a $1 \mathrm{keV}$ optimal filter to "resistance space" pulses at different energies. We notice that neither is the transformed pulse close to the actual TES resistance, nor is the obtained gain scale linear, which indicates that this simple transformation is not suitable for the X-IFU pixels. Although in previous examples of using this transformation the effect of the inductance was very small, ${ }^{13,14}$ in the application considered here, relatively large inductors are used in order to critically damp the pixels and limit the load on the FDM readout scheme. ${ }^{15}$ This however has the effect of producing a more non-linear response and slower current pulses. We further note that because the TES electro-thermal equations are not linear, there is also no reason for the TES resistance signal to be linear with energy in all cases.

The introduction of the inductance derivative term in Equation 8 would significantly increase the high frequency noise. Rather than looking for a more accurate resistance proxy, we therefore looked for a simple transformation, similar to Equation 8, that would produce the desired linear gain scale. Keeping in mind that the optimal filtering process is transparent to any linear modification of the signal, we used a series of simulated templates at different energies to fit the following generic transformation*:

$$
\tilde{R}^{\prime}(t)=\frac{V_{0}}{I_{f i t}+I(t)} .
$$

${ }^{*}$ Equation 8 corresponds to $I_{f i t}=0 \mathrm{~A}$, the subtraction by $R_{L}$ being transparent to the optimal filtering process. 

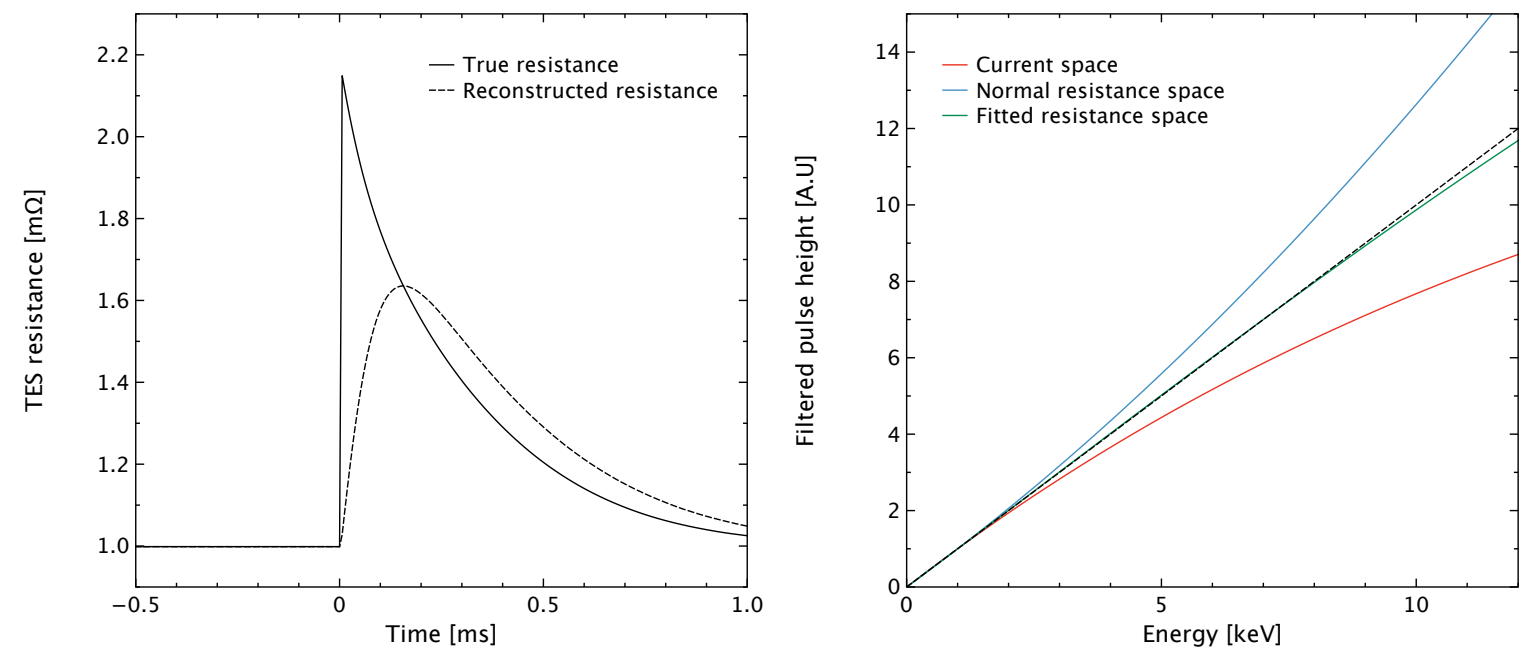

Figure 2. The "resistance space" analysis. Left: Comparison of the "resistance" pulse obtained from the transformation of the measured current pulse with Equation 8 (reconstructed resistance) with the actual simulated resistance at $7 \mathrm{keV}$ (true resistance). Because of the circuit inductance, the current signal is slower than the resistance and this simple transformation does not give a reliable estimate of the TES resistance during the event. Right: Gain scales corresponding to "current space", "normal resistance space" (Equation 8) and "fitted resistance space" optimal filtering (Equation 9). The dotted line shows a perfectly linear scale.

The optimal $I_{f i t}$ was thus found to be $45.3 \mu \mathrm{A}$ (the obtained quasi-linear gain scale is shown in Figure 2, right). In the following sections, for better readability, we refer to this final transformation as "fitted resistance space" and to Equation 8 as "normal resistance space".

\subsection{Covariance based reconstruction}

The aim of using an optimal filter is twofold: weight by the noise power in order to reject potential noise tones coming for instance from the cryo-chain, and use a template matched to the signal to maximize the signal-to-noise of the reconstruction. Because the constant shape assumption is not verified, the filter is not optimized for all energies. A first modification can thus be to use a locally linear assumption instead of a global one.

Let us consider a family of pulse templates $s_{i}(t)$ obtained at the energies $E_{i}^{\dagger}$. The signal of an event whose energy is straddled by $E_{\alpha}$ and $E_{\beta}$ can be approximated as:

$$
d(t)=b+s_{\alpha}(t)+\frac{E-E_{\alpha}}{E_{\beta}-E_{\alpha}}\left(s_{\beta}(t)-s_{\alpha}(t)\right),
$$

where $b$ is the baseline level that will be rejected by the processing.

On top of the linear assumption, by using a simple noise power spectrum to characterize the noise properties during the pulses, the optimal filtering further implicitly assumes that the noise is stationary. This is however known not to be true, the Johnson noise decreasing during the pulse as the resistance increases. ${ }^{10,13,16} \mathrm{~A}$ more complete representation of the noise during the course of the pulse is the noise covariance matrix measured on top of the pulses (see Fig. 3) and its inverse can be used instead of the noise power to weight the optimization problem. ${ }^{17-19}$ If we take the first order expansion of the pulse shape (see Eq. 10) as well as the constraint of rejecting the baseline level $b$, we can define a new $\chi^{2}$ problem as:

$$
\begin{aligned}
\chi^{2} & =\left\|d(t)-\left[s_{\alpha}(t)-\frac{E_{\alpha}}{E_{\beta}-E_{\alpha}}\left(s_{\beta}(t)-s_{\alpha}(t)\right)\right]-E \times \frac{s_{\beta}(t)-s_{\alpha}(t)}{E_{\beta}-E_{\alpha}}-b\right\|_{C^{-1}}^{2}, \\
& =\left(D^{\prime}-E \times S^{\prime}-b\right)^{T} C^{-1}\left(D^{\prime}-E \times S^{\prime}-b\right) \text { using a matrix representation, }
\end{aligned}
$$

${ }^{\dagger}$ In the case of our simulations, this was obtained by averaging many signals simulated at the desired energy. 

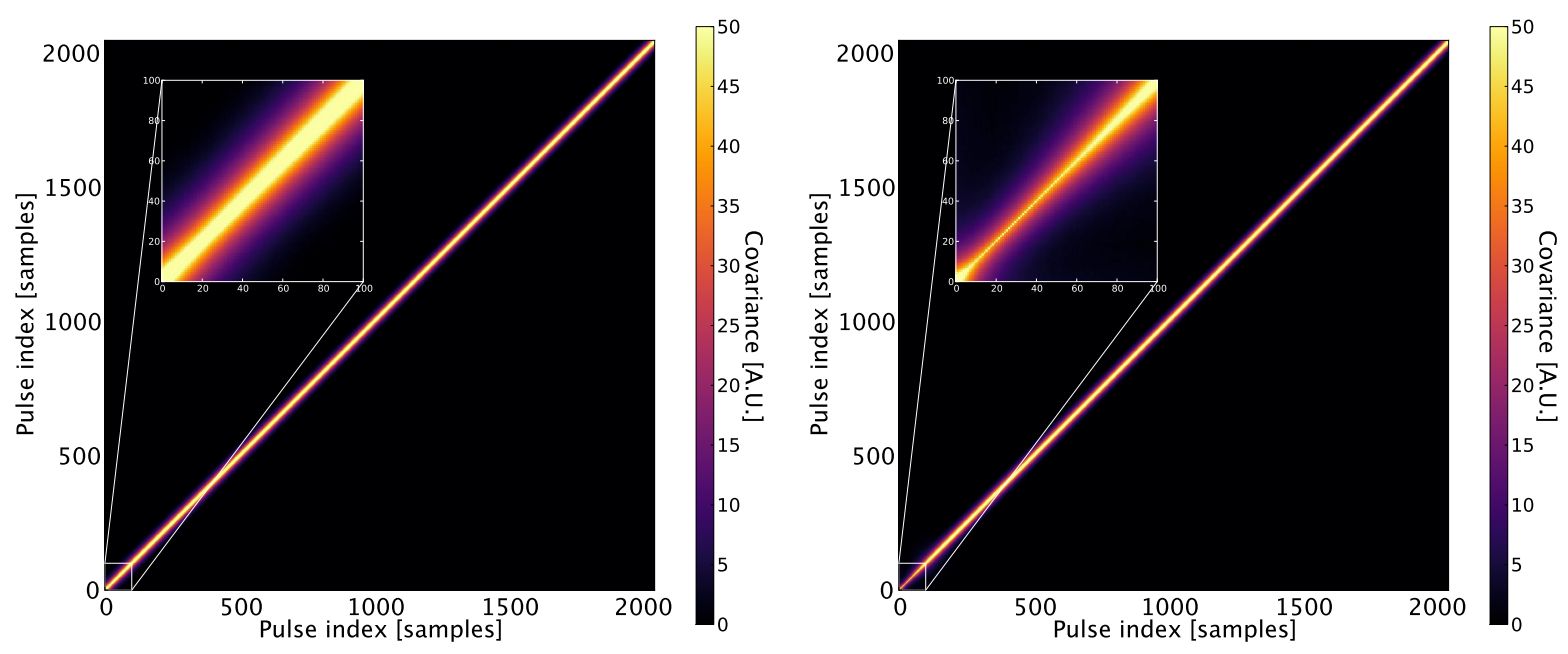

Figure 3. Covariance matrices measured from simulated X-IFU pulses at 1 (left) and $7 \mathrm{keV}$ (right). The insets show the region corresponding to the top of the pulses. Because the pulses were simulated in phase with the readout process, the matrices are mostly diagonal and do not show the usual pattern near the pulse arrival time. One can notice the non-stationary character of the noise through the variable amplitude across the diagonal, notably in the inset at $7 \mathrm{keV}$.

with $E$ and $b$ being the fitted parameters, $d^{\prime}(t)=d(t)-\left[s_{\alpha}(t)-E_{\alpha}\left(s_{\beta}(t)-s_{\alpha}(t)\right) /\left(E_{\beta}-E_{\alpha}\right)\right]$ and $s^{\prime}(t)=$ $\left(s_{\beta}(t)-s_{\alpha}(t)\right) /\left(E_{\beta}-E_{\alpha}\right)$. From there, we can build a new estimator $\hat{\theta}=[\hat{E}, \hat{b}]$ :

$$
\hat{\theta}=\left(R^{T} C^{-1} R\right)^{-1} R^{T} C^{-1} D^{\prime} \text { with } R=\left[S^{\prime}, \mathbb{1}\right] .
$$

This simple formula can only be used if a constant covariance matrix is assumed in the $\alpha-\beta$ interval. In the work presented here, we took the average of the matrices measured at the two energies. It is also possible to linearly interpolate between the two calibration points and obtain a more precise estimation at the cost of a much more computationally intensive formulation, which was deemed to be prohibitive in the case of a future on-board implementation. ${ }^{18}$ Contrary to the others, this reconstruction method also uses a known baseline and was therefore only kept as a reference of the achievable performance in terms of energy resolution degradation with energy and will be referred to as "interpolated covariance analysis". This derivation presented here is also only applicable under the assumption of the knowledge of the two calibration points straddling the pulse. This can however usually be achieved by using a very crude estimation of the energy, like e.g. the raw pulse height.

\section{PERFORMANCE COMPARISON OF THE DIFFERENT TECHNIQUES}

The performance of a pulse reconstruction technique can mainly be separated into two categories:

- its capacity to compensate for the detector's non-linearity and have a limited degradation of the energy resolution with energy,

- and its capacity to retain good energy resolution at high count rates.

\subsection{Energy resolution degradation with energy}

We simulated with tessim a large set of isolated pulses at different energies ranging from 0.2 to $8 \mathrm{keV}$ and reconstructed it with the different techniques. The energy resolution as a function of energy is then simply estimated from the scatter of the measured values. In order to free the analysis from record length effects ${ }^{20} \mathrm{a}$ large record length of $\sim 13 \mathrm{~ms}$ (2048 samples) was used. Figure 4 shows the results of our analysis, comparing the performance of the different pulse processing methods. All values are after gain scale correction (see Sect. 3.1) and perfect knowledge was assumed by calibrating each method with a very large number of pulses (see Sect. 6 for the differential effect of calibration). 


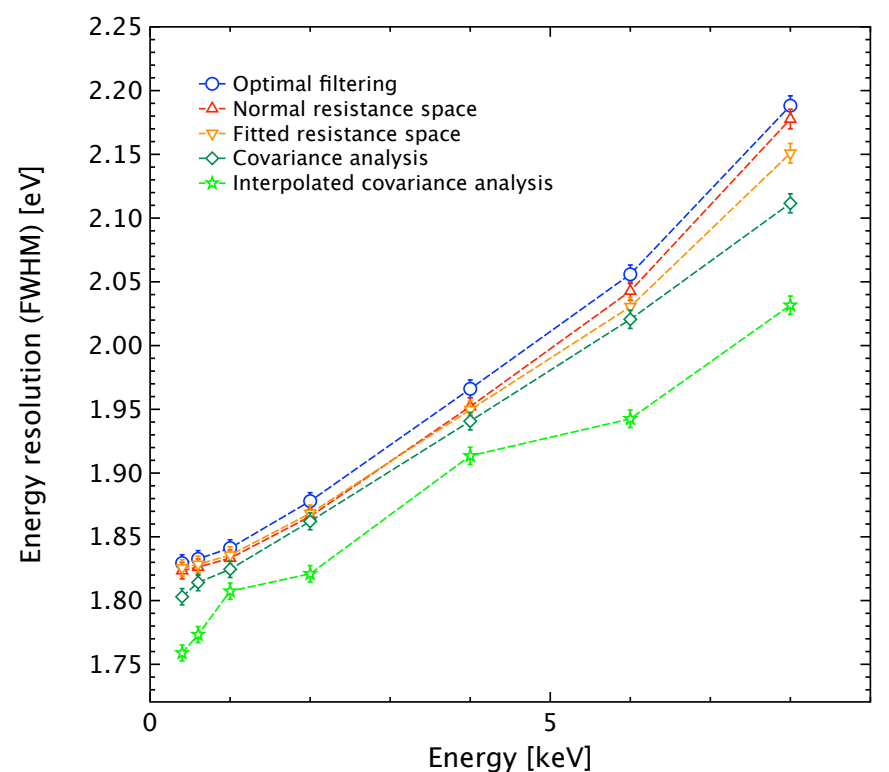

Figure 4. Comparison of the energy resolution performance of the different pulse reconstruction techniques as a function of energy for the baseline X-IFU pixels. The error bars give the $1 \sigma$ uncertainty from the simulations.

Overall, all techniques show good linearity properties with an energy resolution of $\sim 2.1 \mathrm{eV}$ at $7 \mathrm{keV}$ starting from a low-energy resolution of $\sim 1.82 \mathrm{eV}$. The covariance analysis shows a systematic improvement of $\sim 0.03-$ $0.04 \mathrm{eV}(\sim 0.5 \mathrm{eV}$ root mean squared) compared to the standard optimal filtering technique and appears in terms of performance as a promising candidate. The improvement from the resistance space analysis is more limited but nonetheless significant above $4-5 \mathrm{keV}$. We note that the modification of the transformation formula did further decrease the degradation at high energies. Comparing to the reference interpolated covariance analysis, we see that there remains some margin for improvement even though at least part of it is due to a known baseline in this case (see Sect. 3.3).

\subsection{Energy resolution degradation with count rate}

At high count rates, the current pulses created by the individual X-ray impacts will start to get closer and closer, leading to two distinct effects on the energy reconstruction of the events: firstly, the signal tail from preceding pulses will bias the energy estimation, and secondly, the presence of subsequent events will limit the amount of data useable for the pulse processing and lead to a degradation of the achievable energy resolution. ${ }^{20}$

To characterize these effects, we simulated multiple series of pulse doublets separated by different fixed pulse distances. The latter pulses were then used to estimate the varying bias of the energy estimation as a function of pulse separation, and the former to measure the energy resolution as a function of record length. As for the non-linearity study, the results presented in Figure 5 were gain scale corrected and perfect calibration knowledge was assumed. This study was performed with monochromatic $7 \mathrm{keV}$ pulses which is the limiting energy for the $2.5 \mathrm{eV}$ resolution requirement and therefore the most stringent situation.

All techniques show a sharp increase of the energy measurement bias below a few ms separation where no proper reconstruction can be performed anymore (see Fig. 5, left). In practice, these pulses would need to be flagged as secondaries and removed from the science data. ${ }^{6}$ Some recent studies have tried to characterize and correct for this bias, ${ }^{21}$ or simultaneously fit several pulses ${ }^{22}$ to get the best throughput and energy resolution compromise at high count rates. However, as this bias depends on both the time separation and the energy, the applicability to such methods on the non-linear X-IFU pulses over the full instrument bandwidth remains unclear.

Concerning the energy resolution degradation with record length, as could be expected, the covariance based analysis provides an improvement to the standard optimal filtering based techniques. This method takes into 

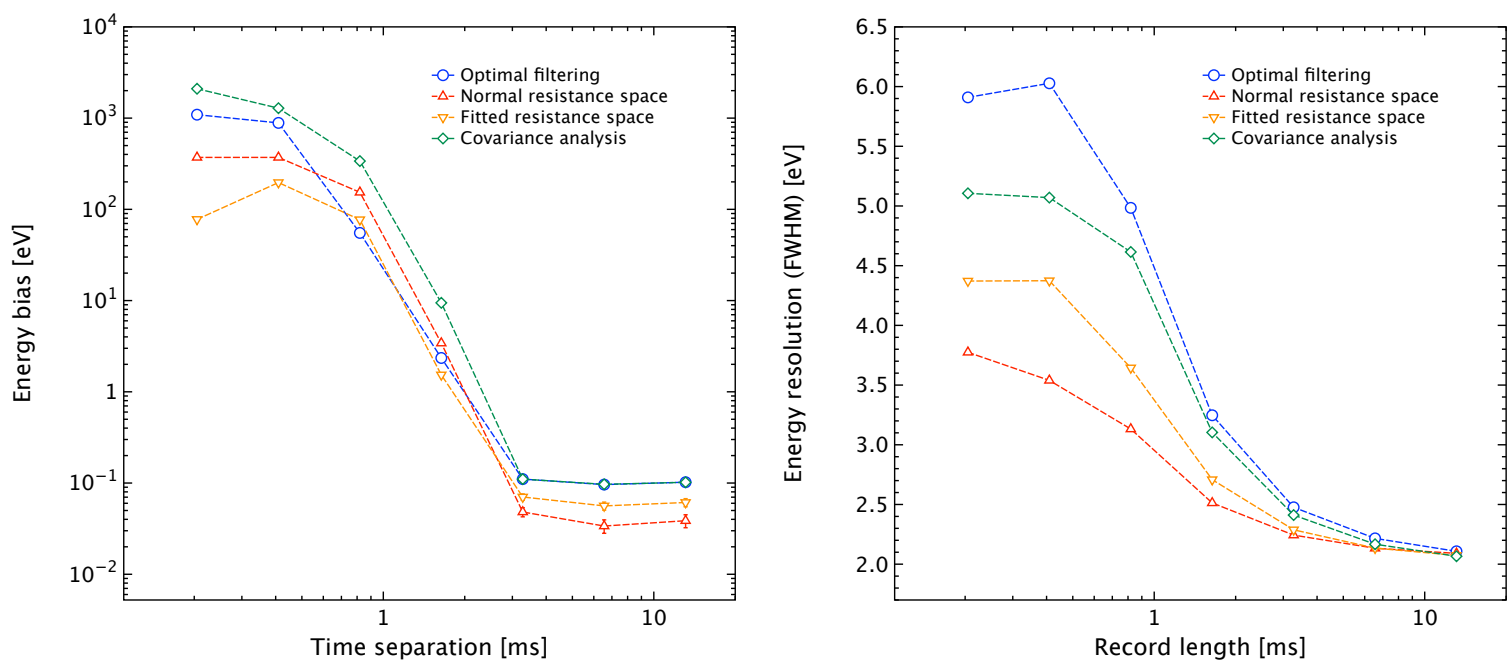

Figure 5. Comparative count rate performance of the different pulse reconstruction techniques for the baseline X-IFU pixels. Left: Energy bias as a function of time separation since the previous event. Right: Energy resolution (FWHM) as a function of record length.

account the non-stationarity character of the noise during the pulse and therefore makes the best out of the few available data points. More surprisingly, the resistance space techniques show even better performance. This is actually due to the ratios of the pulse and noise powers at different frequencies having been changed, resulting in a higher pixel effective roll-off frequency and therefore a slower degradation with record length. ${ }^{13,20}$ If the covariance analysis shows the best performance for large record lengths, the resistance space methods could therefore be preferred for the treatment of pulses with limited record lengths. We note that this application would only concern the observation of bright point sources which are not the most privileged targets for the $\mathrm{X}-\mathrm{IFU}$ and would mostly offer an improvement to the observatory capability of the instrument.

\section{COMPUTATIONAL COST ESTIMATES OF THE DIFFERENT METHODS}

Whereas, the overall performance of the reconstruction techniques should be one of the main drivers for the final choice of the algorithm, the fact that a limited computational power will be available on board cannot be overlooked. In this section we will therefore briefly discuss the computational cost of each pulse processing solution. The aim is not to present here accurate estimates of the maximum processable pulse rate for a realistic space qualified CPU and every reconstruction technique, which would be at best a wild guess in this early phase, but rather to highlight the differences/similarities in terms of complexity in our intention to propose an as complete as possible view of the problem. Table 2 gives, for the methods described in Section 3 the number of each type of operation required to process one high resolution pulse (due to the X-IFU count rate requirement of $80 \%$ high resolution throughput at $1 \mathrm{mCrab}$, the high resolution record length is limited to 1024 samples) in pure algorithmic terms. We note that we do not consider here any triggering stage as it is independent of the reconstruction choice. In all cases, we considered that each pulse would need to be filtered 3 times (with \pm 1 sample offset) in order to correct for sub-sample arrival time jitter.

As previously mentioned, the optimal filtering can be summarized to a single dot product and is therefore the most efficient solution. For the covariance analysis, only an additional subtraction by a pre-computed template is actually necessary. We would like to emphasize the fact that even if Equation 13 shows multiple matrix products, all the first terms can actually be pre-multiplied and the energy estimation is again only a dot product ${ }^{\ddagger}$. For

${ }^{\ddagger}$ Equation 13 gives the formula to estimate both the energy and the baseline level at the same time. Even if this level could be required to correct for long term variations, it would also need to be computed for the other techniques or could be done by another process (e.g. baseline monitoring at the triggering stage). If this estimation is discarded, the energy estimation collapses to a single dot product. 
Table 2. Computational cost of the different reconstruction techniques to process one high resolution event of 1024 samples.

\begin{tabular}{|l|l|l|l|}
\hline Reconstruction method & Nb. of additions & Nb. of multiplications & Nb. of divisions \\
\hline Optimal filtering & 3072 & 3072 & 0 \\
Normal resistance space & $3072^{b}$ & 3072 & 1024 \\
Fitted resistance space & $4096^{b}$ & 3072 & 1024 \\
Covariance analysis & $4096(+1024)^{a}$ & $3072(+1024)^{a}$ & 0 \\
\hline
\end{tabular}

Notes $-{ }^{a}$ The covariance analysis method relies on the identification of the two calibration points straddling the analyzed pulse. The first rough estimation of the energy (whose cost was neglected here) might therefore not directly give the correct interval, in which case an additional filtering might be performed. This would however only happen for events of energy very close to the calibration points, i.e. very rarely. ${ }^{b}$ As mentioned in Sect. 3.2, optimal filtering is transparent to any additional linear transformation of the signal. In Equation 8, the load resistance term can therefore be omitted and the transformation becomes a simple division. This can however not be done in the "fitted resistance space" case.

the resistance space analysis, the difference comes from the pre-transformation of the signal, which makes it the heaviest method. We note that all the methods presented here nonetheless require very limited computational power.

\section{CALIBRATION ISSUES}

The last aspect that should be looked at when comparing reconstruction techniques is calibration. Whereas this is usually not an issue when studying data coming from a few pixels for which a large number of sample pulses can be relatively quickly obtained, it could become a critical aspect for the X-IFU and its 3840 pixels, if they need to be independently characterized. To investigate the different needs of the pulse processing methods, we simulated calibration processes using a limited amount of pulses and compared the performance degradation observed at $7 \mathrm{keV}$. The covariance analysis was set up using 6,7 , and $8 \mathrm{keV}$ monochromatic simulations, while all the optimal filtering methods used filters at $1 \mathrm{keV}$. For a more meaningful comparison, like for the covariance analysis, we also used the noise residuals on top of the pulses instead of empty streams to measure the noise power spectra needed by the optimal filters.

Figure 6 shows the results of our comparison. As can be seen, all optimal filtering techniques actually need only very few pulses $(\sim 1000)$ to reach the final energy resolution. We however need to keep in mind that with real non-monochromatic X-ray sources, more pulses will be needed to obtain stable pulse templates at each energy than what we observed here. The covariance analysis is much more demanding ( 400000 pulses, if not more) and if the X-IFU pixels turn out to require independent calibration, this could well disqualify this reconstruction method. This huge difference with the other methods is actually due to the estimation of the noise covariance matrices which suffer from much poorer statistics compared to simple power spectra computations.

\section{CONCLUSION}

We have presented here an extended benchmark of the different pulse reconstruction techniques that could be considered for implementation in the on-board X-IFU Event Processor. Overall, all techniques show very similar performances, with the covariance analysis ${ }^{17-19}$ showing the most promising improvement of the energy resolution degradation with energy compared to standard optimal filtering $(\sim 0.04 \mathrm{eV}$ or $\sim 0.5 \mathrm{eV}$ root mean squared at $7 \mathrm{keV}$ ). This method however requires a significantly more demanding calibration process than the others and this may prevent its implementation for the X-IFU. On the other hand, resistance space analysis could become a strong candidate. It indeed provides some performance improvement $(\sim 0.02 \mathrm{eV}$ or $\sim 0.2$ root mean squared at $7 \mathrm{keV}$ ) at a very limited computational cost and no impact on the calibration process. It is also the most robust technique at high count rates.

We note that the simulations performed here were all done in a fixed environment for the TES and no low frequency variations of for instance the bath temperature or the voltage bias were considered. This will introduce degradations of the energy resolution which in principle might be different depending on the used reconstruction 


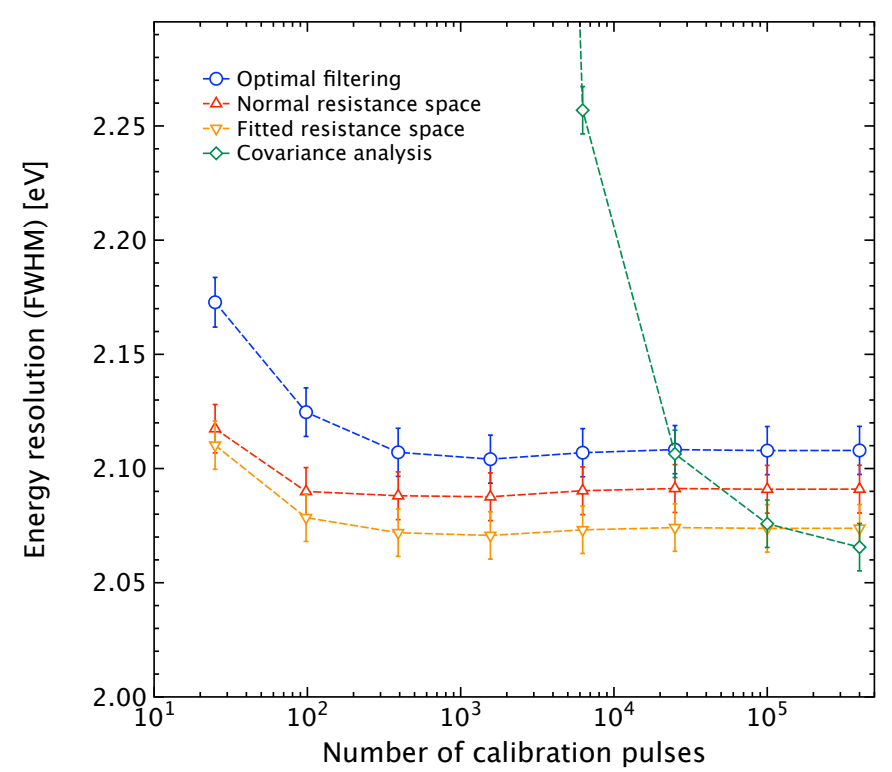

Figure 6. Comparison of the calibration needs for the different reconstruction methods with the baseline X-IFU pixels. The achieved energy resolution at $7 \mathrm{keV}$ is given as a function of the number of pulses used for the calibration. The error bars give the $1 \sigma$ uncertainty from the simulation statistics. As can be seen, the covariance analysis requires much more data than the optimal filtering techniques. The differences at high pulse calibration numbers is due to the energy dependent energy resolution (see Sect. 4) and is not related to the calibration process.

technique. To tackle this issue, a new processing technique based on Principal Component Analysis was also proposed which showed promising results for narrow band studies. ${ }^{23,24}$ Its application to continuum spectra using an automated calibration process however needs to be investigated.

We further note that this study was conducted for particularly linear TESs which explains why only limited differences can be found between the different techniques. This proved to be also true for lower heat capacity TESs considered for implementation in a dedicated high count rate subarray of the X-IFU and for which very similar results were found (see Appendix A). Larger improvements could be observed in other types of detectors but we do not exclude that the ordering of the different reconstruction methods in terms of performance might also be different. The extension to severely non-linear detectors, including those operated up to their saturation level, would however need to be carefully investigated using notably a more realistic modeling of the superconducting transition.

The above results were obtained using the SIXTE simulation package, thus demonstrating its unique capabilities to assess the instrument performance under different configurations, as required in the early phases of the overall instrument optimization.

\section{APPENDIX A. APPLICABILITY TO THE X-IFU SMALL PIXEL ARRAY}

The baseline configuration for the X-IFU TES array consists of an assembly of 3840 identical pixels. Other sensor array configurations are however being considered with TESs of different properties in attempting to improve the $\mathrm{X}$-IFU performance in terms of field of view, count rate performance, and potentially spectral resolution. These configurations notably include at their center a small pixel array (SPA) dedicated to the observation of bright point sources. To populate the SPA, smaller heat capacity pixels are considered, based on recent developments for Solar physics application. ${ }^{25}$ In this appendix, we extended our analysis to this other pixel design whose parameters are given in Table 3. We note that in this case, an unexplained noise component was added in the simulations, parametrized by a scaling factor $M$ with respect to the standard Johnson noise. 
Table 3. Pixel parameters used to simulate the X-IFU SPA pixels.

\begin{tabular}{|l|l|}
\hline Pixel parameter & Value \\
\hline Heat capacity at bias $C$ & $0.26 \mathrm{pJ} / \mathrm{K}$ \\
Bath conductance at bias & $300 \mathrm{pW} / \mathrm{K}$ \\
Heat bath power flow exponent & 4 \\
$\alpha$ & 100 \\
$\beta$ & 10 \\
Unexplained noise factor $M$ & 0.8 \\
Bias resistance $R_{0}$ & $1.1 \mathrm{~m} \Omega$ \\
Bias temperature $T_{0}$ & $90 \mathrm{mK}$ \\
Bias current $I_{0}$ & $72.6 \mu \mathrm{A}$ \\
Effective load resistance $R_{L}$ & $91 \mu \Omega$ \\
Effective circuit inductance $L$ & $122 \mathrm{nH}$ \\
Read-out sampling frequency & $156.25 \mathrm{kHz}$ \\
\hline
\end{tabular}

Figure 7 shows the results of our simulations for the degradation of the energy resolution with energy. We find very similar results to those of the baseline pixels with the covariance analysis being the best reconstruction method. The "fitted resistance space" analysis again offers a smaller improvement but in this case, the "normal resistance space" reconstruction increases the energy uncertainty. This further indicates that this transformation is not directly applicable to high inductance cases. Overall, we however note that the differences between the methods are more limited than for the standard X-IFU pixels, including with the reference "interpolated covariance analysis".

In terms of performance at higher count rates (see Figure 8), as could be expected, these pixels, optimized for higher count rates, show slower performance degradation for all reconstruction techniques. The qualitative behavior nonetheless remains the same as before with a very sharp increase of the energy measurement bias when the previous pulse is too close, and the "normal resistance space" analysis showing the best performance for low record lengths.

Overall, the similarity of our results for both pixel types seems to indicate that the performance comparison presented here could be extended to a wider range of detectors.

\section{ACKNOWLEDGMENTS}

We thank Harvey Moseley and Dale Fixsen for helpful discussions on covariance reconstructions and principal component analysis. X. Barcons, M.T. Ceballos and B. Cobo acknowledge financial support from Spanish Ministry of Economy and Competitiveness (MINECO) under grant ESP2014-53672-C3-1-P, co-funded by FEDER funds. 


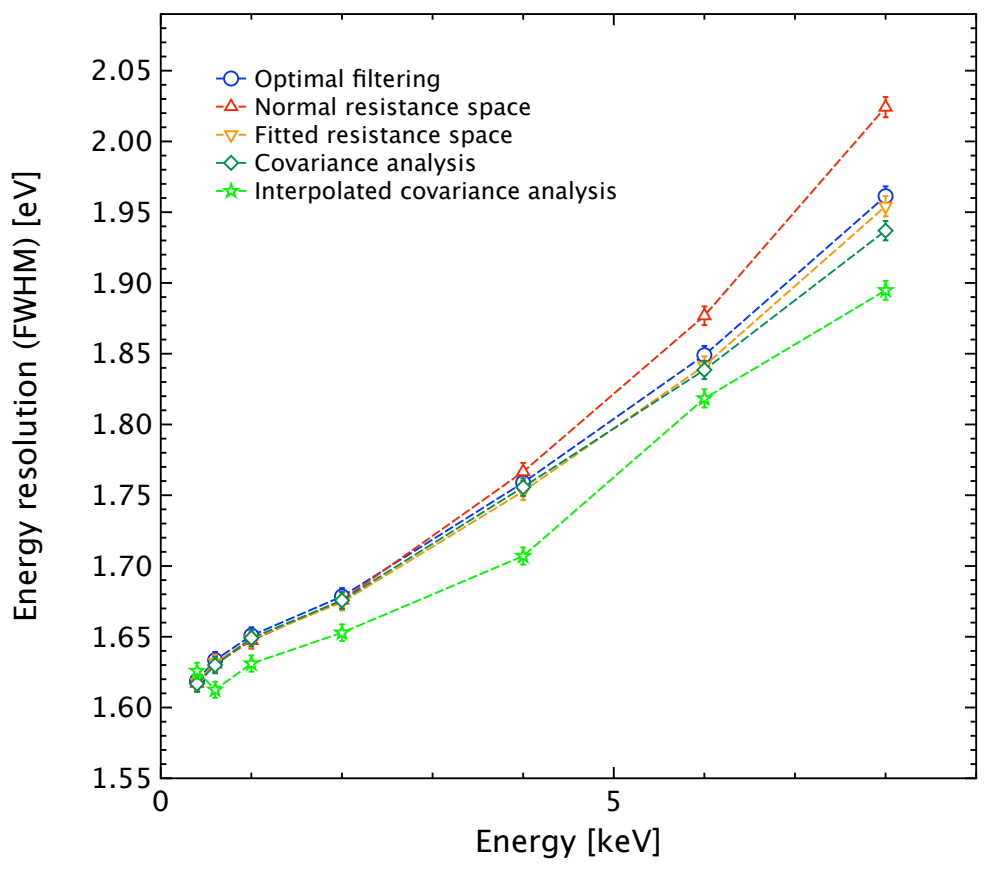

Figure 7. Comparison of the energy resolution performance of the different pulse reconstruction techniques as a function of energy for the X-IFU SPA pixels. The error bars give the $1 \sigma$ uncertainty from the simulations.
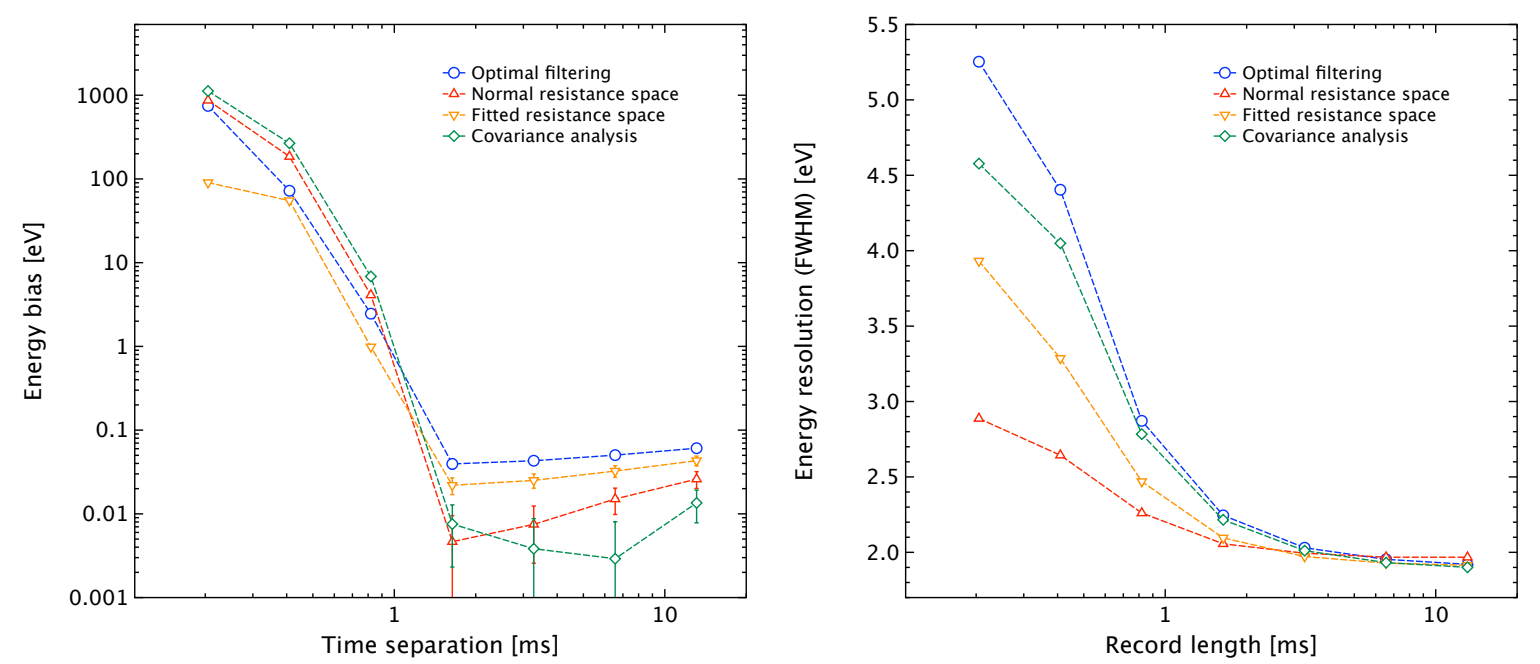

Figure 8. Comparative count rate performance of the different pulse reconstruction techniques for the X-IFU SPA pixels. Left: Energy bias as a function time separation since the previous event. Right: Energy resolution (FWHM) as a function of record length. The error bars give the $1 \sigma$ uncertainty from the simulations. 


\section{REFERENCES}

[1] Ravera, L., Barret, D., den Herder, J. W., Piro, L., Clédassou, R., Pointecouteau, E., Peille, P., Pajot, F., Arnaud, M., Pigot, C., Duband, L., Cara, C., den Hartog, R. H., Gottardi, L., Akamatsu, H., van der Kuur, J., van Weers, H. J., de Plaa, J., Macculi, C., Lotti, S., Torrioli, G., Gatti, F., Valenziano, L., Barbera, M., Barcons, X., Ceballos, M. T., Fàbrega, L., Mas-Hesse, J. M., Page, M. J., Guttridge, P. R., Willingale, R., Paltani, S., Genolet, L., Bozzo, E., Rauw, G., Renotte, E., Wilms, J., and Schmid, C., "The X-ray Integral Field Unit (X-IFU) for Athena," in [Space Telescopes and Instrumentation 2014: Ultraviolet to Gamma Ray], Proc. SPIE 9144, 91442L (July 2014).

[2] Barret, D., den Herder, J. W., Piro, L., Ravera, L., Den Hartog, R., Macculi, C., Barcons, X., Page, M., Paltani, S., Rauw, G., Wilms, J., Ceballos, M., Duband, L., Gottardi, L., Lotti, S., de Plaa, J., Pointecouteau, E., Schmid, C., Akamatsu, H., Bagliani, D., Bandler, S., Barbera, M., Bastia, P., Biasotti, M., Branco, M., Camon, A., Cara, C., Cobo, B., Colasanti, L., Costa-Kramer, J. L., Corcione, L., Doriese, W., Duval, J. M., Fabrega, L., Gatti, F., de Gerone, M., Guttridge, P., Kelley, R., Kilbourne, C., van der Kuur, J., Mineo, T., Mitsuda, K., Natalucci, L., Ohashi, T., Peille, P., Perinati, E., Pigot, C., Pizzigoni, G., Pobes, C., Porter, F., Renotte, E., Sauvageot, J. L., Sciortino, S., Torrioli, G., Valenziano, L., Willingale, D., de Vries, C., and van Weers, H., "The Hot and Energetic Universe: The X-ray Integral Field Unit (X-IFU) for Athena+," ArXiv e-prints (Aug. 2013).

[3] Ravera, L., Cara, C., Ceballos, M. T., Barcons, X., Barret, D., Clédassou, R., Clénet, A., Cobo, B., Doumayrou, E., den Hartog, R. H., van Leeuwen, B.-J., van Loon, D., Mas-Hesse, J. M., Pigot, C., and Pointecouteau, E., "The DRE: the digital readout electronics for ATHENA X-IFU," in [Space Telescopes and Instrumentation 2014: Ultraviolet to Gamma Ray], Proc. SPIE 9144, 91445T (July 2014).

[4] Szymkowiak, A. E., Kelley, R. L., Moseley, S. H., and Stahle, C. K., "Signal processing for microcalorimeters," Journal of Low Temperature Physics 93(3), 281-285 (1993).

[5] Boyce, K. R., Audley, M. D., Baker, R. G., Dumonthier, J. J., Fujimoto, R., Gendreau, K. C., Ishisaki, Y., Kelley, R. L., Stahle, C. K., Szymkowiak, A. E., and Winkert, G. E., "Design and performance of the ASTRO-E/XRS signal processing system," in [EUV, X-Ray, and Gamma-Ray Instrumentation for Astronomy X], Siegmund, O. H. and Flanagan, K. A., eds., Proc. SPIE 3765, 741-750 (Oct. 1999).

[6] Seta, H., Tashiro, M. S., Ishisaki, Y., Tsujimoto, M., Shimoda, Y., Abe, Y., Yasuda, T., Takeda, S., Asahina, M., Hiyama, Y., Yamaguchi, S., Terada, Y., Boyce, K. R., Porter, F. S., Kilbourne, C. A., Kelley, R. L., Fujimoto, R., Takei, Y., Mitsuda, K., Matsuda, K., and Masukawa, K., "The Digital Processing System for the Soft X-Ray Spectrometer Onboard ASTRO-H - The Design and the Performance -," IEEE Transactions on Nuclear Science 59, 366-372 (Apr. 2012).

[7] Wilms, J., Smith, S. J., Peille, P., Ceballos, M. T., Cobo, B., Dauser, T., Brand, T., den Hartog, R., Bandler, S. R., de Plaa, J., and den Herder, J.-W., "TESSIM: a simulator for the ATHENA X-IFU," in [Space Telescopes and Instrumentation 2016: Ultraviolet to Gamma Ray], Proc. SPIE 9905, 9905-192 (July 2016).

[8] Wilms, J., Brand, T., Barret, D., Beuchert, T., den Herder, J.-W., Kreykenbohm, I., Lotti, S., Meidinger, N., Nandra, K., Peille, P., Piro, L., Rau, A., Schmid, C., Smith, R. K., Tenzer, C., Wille, M., and Willingale, R., "ATHENA end-to-end simulations," in [Space Telescopes and Instrumentation 2014: Ultraviolet to Gamma Ray], Proc. SPIE 9144, 91445X (July 2014).

[9] Mather, J. C., "Bolometer noise: nonequilibrium theory," Appl. Opt. 21, 1125-1129 (Mar 1982).

[10] Irwin, K. D. and Hilton, G. C., [Transition-Edge Sensors], 63, Enss, C. (2005).

[11] Kilbourne, C. A., de Korte, P., Smith, S. J., Hoevers, H., van der Kuur, J., Ezoe, Y., and Ullom, J. N., "Ixo/xms detector trade-off study," tech. rep., NASA GSFC (2010).

[12] Smith, S. J., Adams, J. S., Bandler, S. R., Betancourt-Martinez, J. L., Chervenak, J. A., Chiao, M., Datesman, A. M., Eckart, M. E., Ewin, A. J., Finkbeiner, F. M., Kelley, R. L., Kilbourne, C. A., Porter, F. S., Sadleir, J. E., Wassel, E. J., Yoon, W., Bennett, D. A., Doriese, W. B., Hilton, G. C., Swetz, D. S., Ullom, J. N., Akamatsu, H., Gottardi, L., den Hartog, R. H., Jackson, B. D., van der Kuur, J., Barret, D., and Peille, P., "TES pixel parameter design of the microcalorimeter array for the x-ray integral field unit on ATHENA," in [Space Telescopes and Instrumentation 2016: Ultraviolet to Gamma Ray], Proc. SPIE 9905, 9905-85 (July 2016). 
[13] Bandler, S. R., Figueroa-Feliciano, E., Iyomoto, N., Kelley, R. L., Kilbourne, C. A., Murphy, K. D., Porter, F. S., Saab, T., and Sadleir, J., "Non-linear effects in transition edge sensors for X-ray detection," Nuclear Instruments and Methods in Physics Research A 559, 817-819 (Apr. 2006).

[14] Lee, S. J., Adams, J. S., Bandler, S. R., Chervenak, J. A., Eckart, M. E., Finkbeiner, F. M., Kelley, R. L., Kilbourne, C. A., Porter, F. S., Sadleir, J. E., Smith, S. J., and Wassell, E. J., "Fine pitch transition-edge sensor x-ray microcalorimeters with sub-ev energy resolution at $1.5 \mathrm{kev}$," Applied Physics Letters 107(22) (2015).

[15] den Hartog, R., Barret, D., Gottardi, L., den Herder, J.-W., Jackson, B., de Korte, P., van der Kuur, J., van Leeuwen, B.-J., van Loon, D., Nieuwenhuizen, A., and Ravera, L., "Requirements for the detectors and read-out of ATHENA X-IFU," in [Space Telescopes and Instrumentation 2014: Ultraviolet to Gamma Ray], Proc. SPIE 9144, 91445Q (July 2014).

[16] Smith, S. J., Adams, J. S., Bandler, S. R., Busch, S. E., Chervenak, J. A., Eckart, M. E., Finkbeiner, F. M., Kelley, R. L., Kilbourne, C. A., Lee, S. J., Porst, J.-P., Porter, F. S., and Sadleir, J. E., "Characterization of Mo/Au Transition-Edge Sensors with Different Geometric Configurations," Journal of Low Temperature Physics 176, 356-362 (Aug. 2014).

[17] Fixsen, D. J., Moseley, S. H., Cabrera, B., and Figueroa-Feliciano, E., "Optimal fitting of non-linear detector pulses with nonstationary noise," Low Temperature Detectors 605, 339-342 (Feb. 2002).

[18] Fixsen, D., Moseley, S., Cabrera, B., and Figueroa-Feliciano, E., "Pulse estimation in nonlinear detectors with nonstationary noise," Nuclear Instruments and Methods in Physics Research Section A: Accelerators, Spectrometers, Detectors and Associated Equipment 520(1-3), 555 - 558 (2004). Proceedings of the 10th International Workshop on Low Temperature Detectors.

[19] Fixsen, D. J., Moseley, S. H., Gerrits, T., Lita, A. E., and Nam, S. W., "Optimal energy measurement in nonlinear systems: An application of differential geometry," Journal of Low Temperature Physics 176(1), 16-26 (2014).

[20] Doriese, W. B., Adams, J. S., Hilton, G. C., Irwin, K. D., Kilbourne, C. A., Schima, F. J., and Ullom, J. N., "Optimal filtering, record length, and count rate in transition-edge-sensor microcalorimeters," in [American Institute of Physics Conference Series], Young, B., Cabrera, B., and Miller, A., eds., American Institute of Physics Conference Series 1185, 450-453 (Dec. 2009).

[21] Lee, S. J., Bandler, S. R., Busch, S. E., Adams, J. S., Chervenak, J. A., Eckart, M. E., Ewin, A. J., Finkbeiner, F. M., Kelley, R. L., Kilbourne, C. A., Porst, J.-P., Porter, F. S., Sadleir, J. E., Smith, S. J., and Wassel, E. J., "High Count-Rate Studies of Small-Pitch Transition-Edge Sensor X-ray Microcalorimeters," Journal of Low Temperature Physics 176, 597-603 (Aug. 2014).

[22] Fowler, J. W., Alpert, B. K., Doriese, W. B., Fischer, D. A., Jaye, C., Joe, Y. I., O’Neil, G. C., Swetz, D. S., and Ullom, J. N., "Microcalorimeter Spectroscopy at High Pulse Rates: A Multi-pulse Fitting Technique," ApJS 219, 35 (Aug. 2015).

[23] Busch, S. E., Adams, J. S., Bandler, S. R., Chervenak, J. A., Eckart, M. E., Finkbeiner, F. M., Fixsen, D. J., Kelley, R. L., Kilbourne, C. A., Lee, S.-J., Moseley, S. H., Porst, J.-P., Porter, F. S., Sadleir, J. E., and Smith, S. J., "Progress Towards Improved Analysis of TES X-ray Data Using Principal Component Analysis," Journal of Low Temperature Physics (Nov. 2015).

[24] Yan, D., Cecil, T., Gades, L., Jacobsen, C., Madden, T., and Miceli, A., "Processing of X-ray Microcalorimeter Data with Pulse Shape Variation using Principal Component Analysis," Journal of Low Temperature Physics (Jan. 2016).

[25] Smith, S. J., Adams, J. S., Bailey, C. N., Bandler, S. R., Chervenak, J. A., Eckart, M. E., Finkbeiner, F. M., Kelley, R. L., Kilbourne, C. A., Porter, F. S., and Sadleir, J. E., "Small pitch transition-edge sensors with broadband high spectral resolution for solar physics," Journal of Low Temperature Physics 167(3), 168-175 (2012). 